\title{
Design Improvements and Dynamic Characterization on Fluidic Elastomer Actuators for a Soft Robotic Snake
}

\author{
Ming Luo, Weijia Tao, Fuchen Chen, Tri K. Khuu, Selim Ozel, Cagdas D. Onal
}

\begin{abstract}
This paper addresses the design and dynamic analysis of a new generation of fluidic elastomer actuators (FEAs) that offer bidirectional bending developed as motion segments of a pressure-operated soft robotic snake. Our prior work on FEAs has identified a number of limitations, namely a high center of gravity, narrow base, slow dynamics, and a small range of pressure inputs. We developed two versions of FEAs based on an improved design concept with different geometric parameters and characterized their dynamic response under a custom visual tracking system. Compared with the previous actuators, the FEAs developed in this work offer robust operation, safety at larger input pressure values, faster response, lower center of gravity and a flat bottom for better compatibility for snake-like undulatory locomotion.
\end{abstract}

Index Terms-Fluidic elastomer actuator, dynamic characterization, optimal design, soft robotic snake

\section{INTRODUCTION}

Mobile robots offer significant advantages in search and rescue operations [1], [2], [3]. Among alternatives, snake robots stand out due to their simple but effective locomotion mechanism that doesn't rely on complicated limb motions to navigate on rough terrain, on the ground or under water [4], [5]. Snake robots are typically developed as rigid serial kinematic chains with discrete joints approximating the organic undulatory motion of their natural counterpart.

There are several actuation methods employed for rigid snake robots beside the traditional electric motor. For example, [6] uses the pneumatic integrated joint actuators (IJA), which can control position and stiffness. Parallel stage wires and a backbone are adopted in [7] for surgical applications. Ionic polymer metal composites (IPMCs) are used in [8] for a swimming snake robot. A simple snake robot is designed in [9] actuated by shape memory alloys (SMAs) and other support structures. Nevertheless, these approaches have the same fundamental limitation: these actuators could not generate an organic serpentine locomotion because they are based on rigid kinematic arrangements. The resulting snake robots approximate the sinusoidal waveforms and serpenoid body shapes via piecewise straight lines.

A soft snake robot, on the other hand, offers additional capabilities such as adapting to narrow or complex passages, therefore reducing obstacle avoidance requirements during

M. Luo is with the Robotics Engineering Program, Worcester Polytechnic Institute, MA 01609, USA and School of Computer and Information, AnQing Normal University, 246011, Anhui, China mluo@wpi . edu

W. Tao, F. Chen, T. K. Khuu, and S. Ozel are with the Robotics Engineering Program, Worcester Polytechnic Institute, MA 01609, USA. \{wtao, fchen, tkkhuu, sozel\}@wpi.edu

C. D. Onal is with the Mechanical Engineering Department and Robotics Engineering Program, Worcester Polytechnic Institute, MA 01609, USA cdonal@wpi.edu

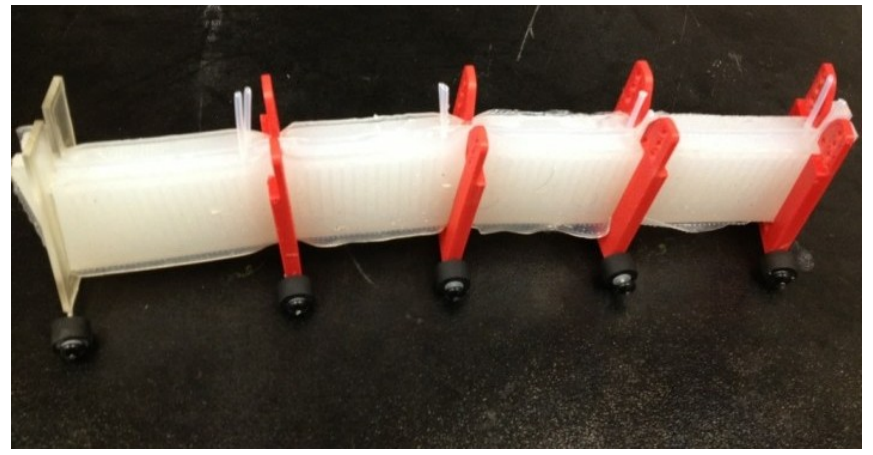

Fig. 1. Experimental prototype of the original design of our pressureoperated soft robotic snake.

locomotion as one of the main utilities of employing a soft body that can produce continuum motions instead of a rigid one.

Soft robotics is an emerging field that has seen a flurry of recent results that may not be achieved with a rigid architecture. A four-legged crawling soft robot was introduced in [10], which also incorporates camouflage capabilities in addition to passing under low passages to adapt to the environment it is operating in. Caterpillar and earthworm-inspired soft robots actuated by shape memory alloys were presented in [11], [12]. Another bio-inspired soft mobile robot is a robotic octopus [13] driven by embedded muscle-like actuators. In prior work, we demonstrated pneumatic actuation of embedded fluidic chambers in an elastomer substrate called the fluidic elastomer actuators (FEAs) as a modular actuation method compatible with soft robotics [14]. We developed electropermanent magnet valves [15] and on-board chemical pressure generation [16] to enable autonomous operation of soft robots with our fluidic actuation principle.

By a serial composition of bidirectional bending FEA segments we first introduced a pressure-operated soft robotic snake in [14], investigated gait algorithms to enable autonomous serpentine locomotion in [17] and developed an accurate theoretical dynamic model of the snake in [18]. The soft snake robot is able to move at approximately $40 \mathrm{~mm} / \mathrm{s}$ under 5 psi pressure input with a coordinated actuation of its segments, with the natural advantage of generating smoother movements than its rigid counterparts [18]. The latest version of our soft robotic snake can be seen in Figure 1.

We based the dynamic model of the soft snake robot [18] on rigid snake robot modeling approaches [19], [20], [21] with modifications to incorporate long bending joints with a constant-curvature assumption. In other words, the 
TABLE I

DIMENSIONS OF THE INVESTIGATED ACTUATORS

\begin{tabular}{l||ccc} 
& Length $[\mathrm{mm}]$ & Width $[\mathrm{mm}]$ & Height $[\mathrm{mm}]$ \\
\hline \hline 1: Thick & 60 & 25 & 30 \\
2: Thin & 110 & 13 & 15 \\
3: Original & 52 & 6 & 33 \\
\hline
\end{tabular}

fundamental difference between our soft snake model and its rigid counterpart is that we treat the whole FEA segment as a joint changing curvature, compared to servo-motor driven pin joints changing angle connected to long links of rigid kinematic chains.

The current body design of the soft robotic snake is not optimal for serpentine locomotion and poses open research problems. The segments are tall and narrow, causing potential balance issues. The fluidic channels embedded in the elastomer for actuation are small and intricate, leading to possible blockage during fabrication. The actuators are likely to malfunction for pressure inputs higher than 5 psi. This is due to the radial expansion of the actuator, leading to undesired stress and eventual damage with increased pressure. In addition, the segments exhibit slow dynamics with large time constants resulting in a limit on the frequency of the serpentine gait and consequently, locomotion speed.

To provide a solution to these design problems for the soft robotic snake, we present a new fluidic elastomer actuator in this work, designed specifically for the requirements of undulatory locomotion. Specifications are made to address three issues: the new design should have (1) a wide base, (2) stronger fluidic channels that can resist pressures larger than 5 psi, (3) accessible, fast, and convenient manufacturing process, and (4) reduced undesired deformations. To meet all of the specifications, the cross section of the actuators are modified to a semi-circular shape with a large base. A thread is tied around the actuator to limit radial deformations [22] and increase material strength. The thin and intricate channels are replaced with a large chamber.

We manufactured two versions of the new FEA design, differing in body length to width ratios: thick-and-short and a thin-and-long design to characterize their static and dynamic responses as well as endurance under relatively high pressures in comparison to the original FEA design. The pressure-operated soft actuators that are presented in this paper can be expanded for general soft robotics research in different areas such as artificial muscles and soft manipulators due to their accessibility.

The outline of the paper is as follows: Section II describes the mechanical design and fabrication process of the three fluidic elastomer actuators in detail. Section III explains the experimental setup, results, and analyses of the data. Section IV concludes the paper and discusses potential future research directions.

\section{Design and Fabrication Process of Next GENERATION FLUIDIC Elastomer ACTUATORS}

This section details the design and manufacturing of the new soft actuators in comparison to the previous iteration.

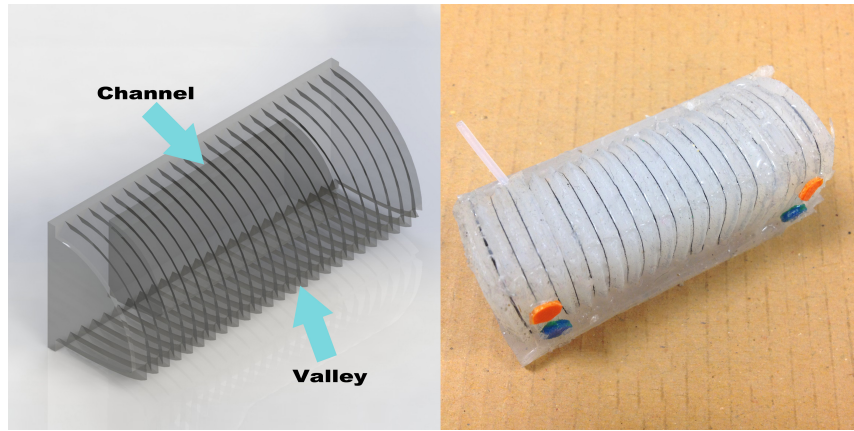

(a)

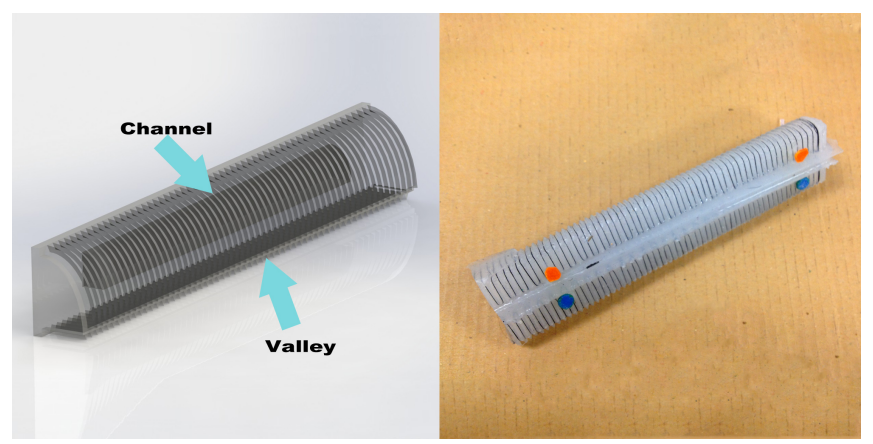

(b)

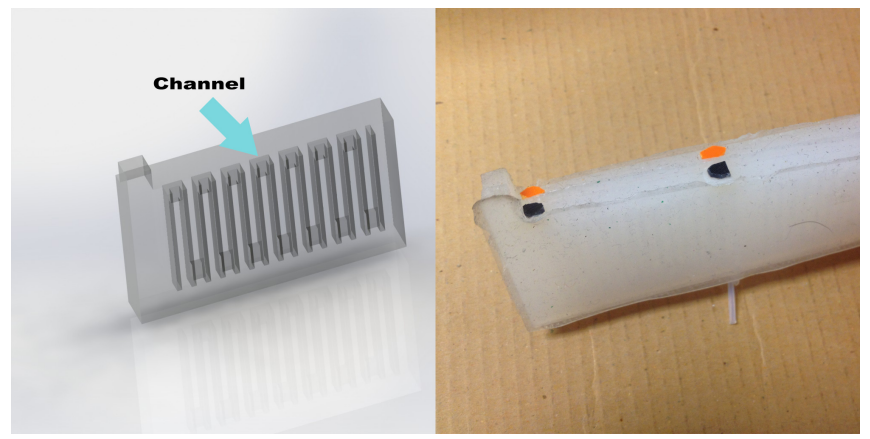

(c)

Fig. 2. Three different kinds of fluidic elastomer actuators were tested: (a) new design: thick and short, (b) new design: thin and long, and (c), the original design.

The original design of the fluidic elastomer actuator is shown in Figure 2(c), with a CAD drawing and experimental prototype. Similarly, Figures 2(a) and (b) display the new FEAs introduced in this paper on two instantiations of the same conceptual design with different length-to-width aspect ratios: a thick and a thin version, respectively. The dimensions of all three actuators analyzed in this paper are listed in Table I. Both actuators have a semi-circular cross-section that is inspired by the body of a natural snake. This design approach also enables the bottom of the actuators to have a larger area compared to the original rectangular design.

In addition, the new designs incorporate soft serrations (series of triangular valleys) on the outside in the longitudinal direction, which provide guides for threads to be tied around the actuator with high repeatability. The threads can be seen as black lines that run parallel around the new actuators at 


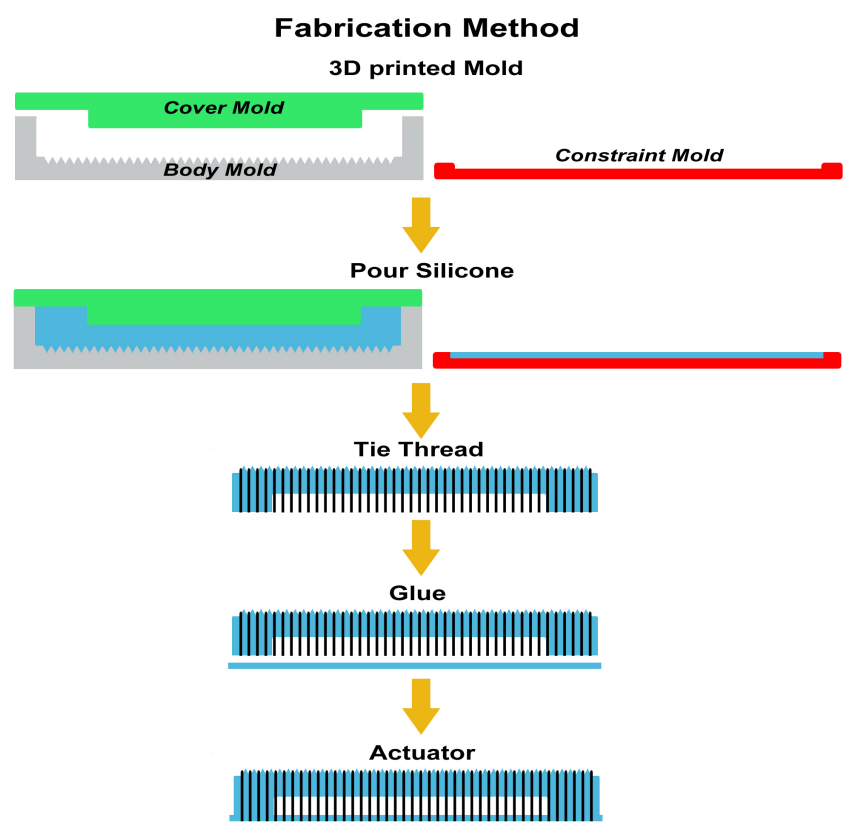

Fig. 3. Fabrication process of the soft actuators.

the right column of Figures 2(a) and (b). The primary goal of the threads is to place a physical constraint on the lateral expansion of the actuators to improve their strength while not inhibiting their bending deformation.

With the threads keeping the lateral expansion in check, an intricate arrangement of parallel fluidic channels (with solid elastomer in-between) is no longer useful. Hence, the network of channels are replaced by a single larger fluidic chamber in the new design. The difference between a chamber and channels can be seen in 3-D models on the left column of Figure 2. The chambers have the same cross-sectional shape as the actuators themselves, offset to provide elastomeric walls. With a single chamber, instead of many small channels, we found that it is easier to seal the actuators during fabrication and there is a reduced possibility of a rupture.

The fabrication process is similar to the original design [17]. Soft silicone rubber material was used (Smooth-on Ecoflex 0030) to mold the FEAs in parts. We use three 3-D printed re-usable molds: a body mold, a cover mold, and a constraint mold as shown in the top row of Figure 3. The semicircular shape of the soft actuators is created in the body mold, which also embeds the thread serrations. The chamber inside the actuator is created by a boss in the middle of the cover mold. An inextensible but flexible material layer is used in the constraint mold to embed in silicone rubber, so that the actuator is limited to only bending motions. The constraint layer was made of fabric in the original design. In the current design, we use a thin plastic film. Another improvement is making certain that the constraint material is in the middle of the constraint mold during fabrication to ensure that the soft actuator bends the same amount in both directions. Holes are cut out into the plastic sheet by a laser

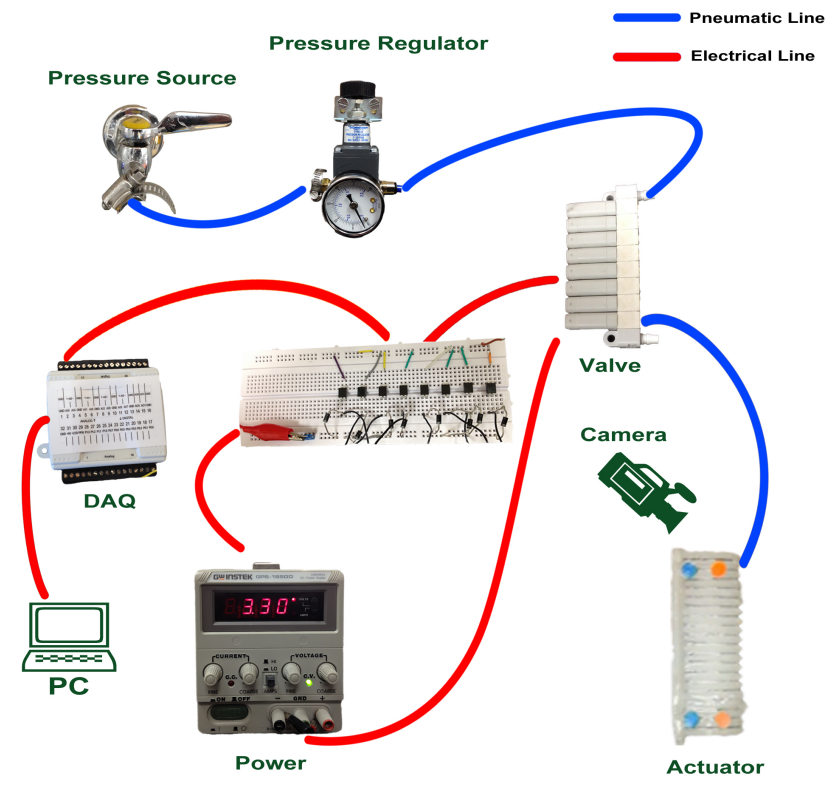

Fig. 4. The experimental system.

cutter. These holes let silicone rubber to flow in and better fix the plastic film after it is curred.

With the mentioned improvements, the entire fabrication process consists of four steps as explained below and illustrated in Figure 3:

Step 1 Silicone rubber is poured in the body mold. Then it is covered by the cover mold. Silicone rubber is poured to constraint mold until halfway. A plastic film is inserted into the constraint mold. Afterwards silicone rubber is poured until the mold is full.

Step 2 Silicone rubber inside the mold takes around 4 hours to cure. Two parts of cured silicone are then removed from the molds.

Step 3 Threads are tied around the soft serrations. Then they are fixed in place using a layer of uncured silicone rubber as glue to make the connection stronger.

Step 4 Silicone rubber body and constraint layer are glued to each other using a thin layer of uncured silicone rubber.

This process takes 8 hours in room temperature, which could be shortened using an oven. Note that these steps describe the fabrication process of a unidirectional actuator. For bidirectional bending, two actuation molds are glued on both sides of the constraint mold.

\section{EXPERIMENTAL RESUlts}

To analyze all three actuators we built an experimental setup composed of a pressurized fluid source, valve drivers, control and data capture system. The whole setup including the soft actuator itself can be seen in Figure 4. A shop air nozzle is used as the pressure source, whose output is filtered through a precise pressure regulator. The typical range of 


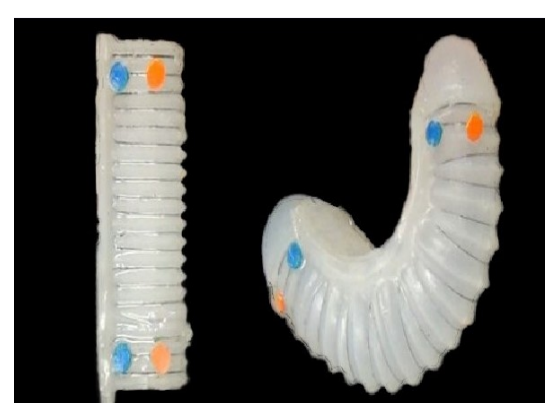

(a) Actuator 1: Thick and Short

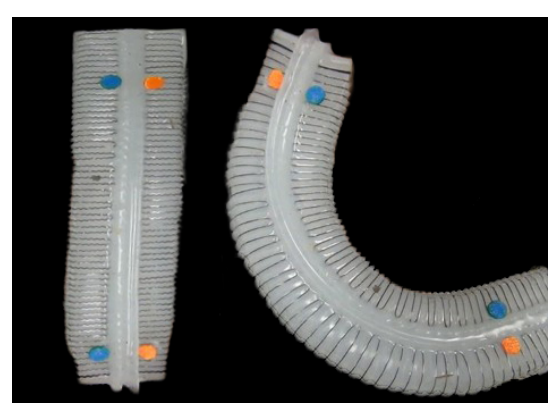

(b) Actuator 2: Thin and Long

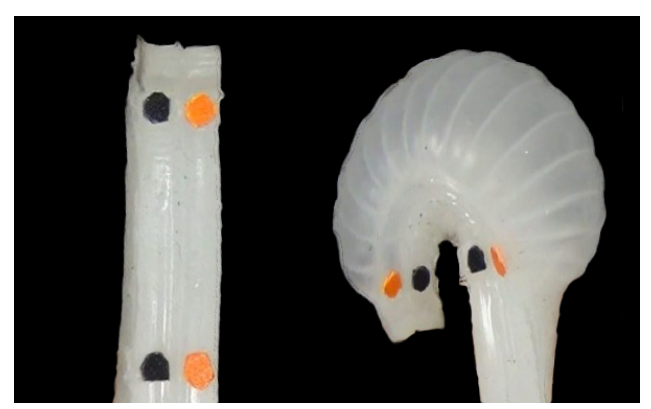

(c) Actuator 3: Original Design

Fig. 5. Bending response of the tested fluidic elastomer actuators for $P_{i n}=5$ psi.

TABLE II

QUALITATIVE COMPARISON OF THREE SOFT ACTUATORS

\begin{tabular}{c||ccc} 
& 1: Thick & 2: Thin & 3: Original \\
\hline \hline Maximum Deflection & Large & Large & Large \\
Response Time & Medium & Small & Large \\
Release Time & Medium & Small & Medium \\
\hline
\end{tabular}

pressure values we used is between 0-10 psi. The regulated pressure is then fed to the actuators through solenoid valves.

A control system is designed to turn the solenoid valves on or off at specified periods. Data acquisition hardware (NI-DAQ PCI 6009) is employed to transfer commands from Matlab to drive each valve. Data Acquisition toolbox is utilized to connect Matlab and NI-DAQ. This board cannot source enough current to drive the valves directly. Therefore, L298N full-bridge drivers are connected to the controller along with a power supply.

The deformation of the fluidic elastomer actuators are monitored using an overhead camera system and tracker software. FEAs bend in a circle arc when pressure is applied. This bending deformation can be described by a single curvature value. Two different-colored markers are positioned on both ends of each actuator (four total). These points are tracked using image processing. The extracted coordinates are then used to calculate the curvature of the actuators in a non-contact manner throughout the experiments presented below.

Before going into quantitative analysis, a qualitative comparison of the dynamic performance of all three soft actuators is listed in Table II and their bending response are displayed in Figure 5 for reference. In this figure, pictures of the investigated actuators are displayed before (left) and after (right) pressure is applied. The large radial deformations, which effectively turn the original design into a balloon under increased pressure inputs, can be seen in Figure 5(c). As expected, these radial deformations were eliminated in the proposed design due to the additional constraint force applied by the surrounding thread.

In the following experiments, the range of input pressure $\left(P_{i n}\right)$ is set to range between 1.25 psi and 9 psi. Pressure step inputs are applied for 7 seconds to observe the dynamic response of each FEA. Figure 6(a-h) displays the change in
TABLE III

THE CHARACTERISTIC PARAMETERS OF EACH ACTUATOR

\begin{tabular}{c||ccc} 
& 1: Thick & 2: Thin & 3: Original \\
\hline \hline$\tau[\mathrm{s}]\left(P_{i n}=5 \mathrm{psi}\right)$ & 0.8781 & $\mathbf{0 . 3 5 2 2}$ & 1.6420 \\
Height of the CoG $[\mathrm{mm}]$ & 12.81 & $\mathbf{8 . 1 8}$ & 16.07 \\
Base width [mm] & $\mathbf{1 0 . 5 1}$ & 5.6 & 3.81 \\
Max pressure [psi] & $\mathbf{9}$ & $\mathbf{9}$ & 5 \\
\hline
\end{tabular}

angle between both ends of the three actuators under various pressure steps. Here note that the original actuator ruptured for a pressure input of $6 \mathrm{psi}$ as previously observed, therefore its response could not be displayed in the second line of Figure 6.

It is stated in the dynamics model of the soft robotic snake, we have described in [18], that amplitude of the serpentine gait has significant influence over the locomotion speed. Therefore, it is more relevant to discuss the response curves in Figure 6 in terms of maximum curvature that can be achieved and the time delay due to dynamic properties of the actuator.

We define the time delay as the difference in time from the moment the valve is turned on until the actuator converges to its static curvature. This is the response time of the actuator. Conversely, the time delay between the valve being turned off and the actuator returning its initial condition is tagged the release time. Both numbers should be as small as possible for optimal functionality, which is very challenging due to physical effects due to the impedance of the fluidic circuit. The fact that the actuation system is driven by external fluidic pressure along with extra tubings also increases the response and release times. These are the parameters along with the maximum deflection listed in Table II to provide a quick comparison of all the significant metrics especially for application as soft segments of a snake robot.

To quantify the dynamic properties of the actuators, we fit the experimental data in Figure 6 to the solution of a first-order differential equation written as:

$$
\kappa=C_{o}+C_{1} e^{-t / \tau},
$$

where $\kappa$ is the curvature of the actuator, $\tau$ is the time constant, $C_{o}$ and $C_{1}$ are constant coefficients. This is a reasonable assumption from circuit equivalence, since the 


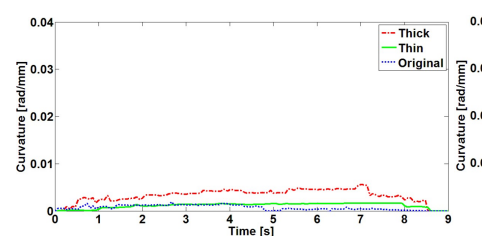

(a) $P_{\text {in }}=1.25 \mathrm{psi}$

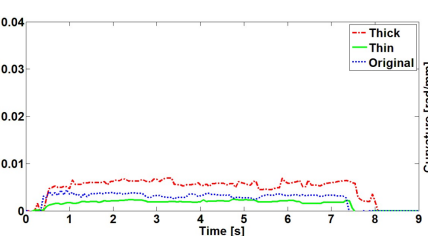

(b) $P_{\text {in }}=2.5 \mathrm{psi}$

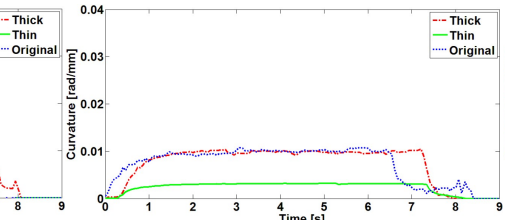

(c) $P_{\text {in }}=3.75 \mathrm{psi}$

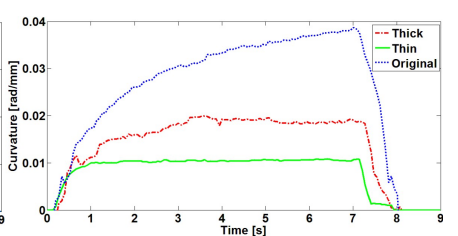

(d) $P_{\text {in }}=5 \mathrm{psi}$

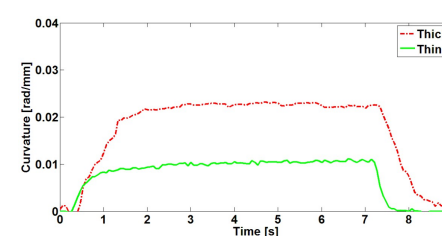

(e) $P_{\text {in }}=6 \mathrm{psi}$

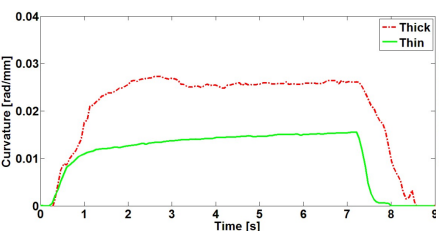

(f) $P_{i n}=6.5 \mathrm{psi}$

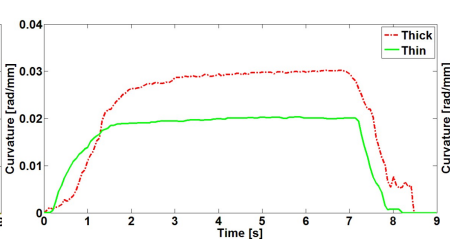

(g) $P_{i n}=7 \mathrm{psi}$

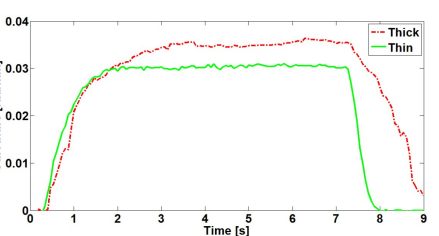

(h) $P_{\text {in }}=8 \mathrm{psi}$

Fig. 6. The shape change in terms of the curvature of the three actuators investigated for step pressure inputs of $1.25 \mathrm{psi}, 2.5 \mathrm{psi}, 3.75 \mathrm{psi}, 5 \mathrm{psi}, 6 \mathrm{psi}$, $6.5 \mathrm{psi}, 7 \mathrm{psi}$, and $8 \mathrm{psi}$. The original fluidic elastomer actuator ruptured at 6 psi. The red, green, and blue curves represent the thick, thin, and original actuators, respectively.

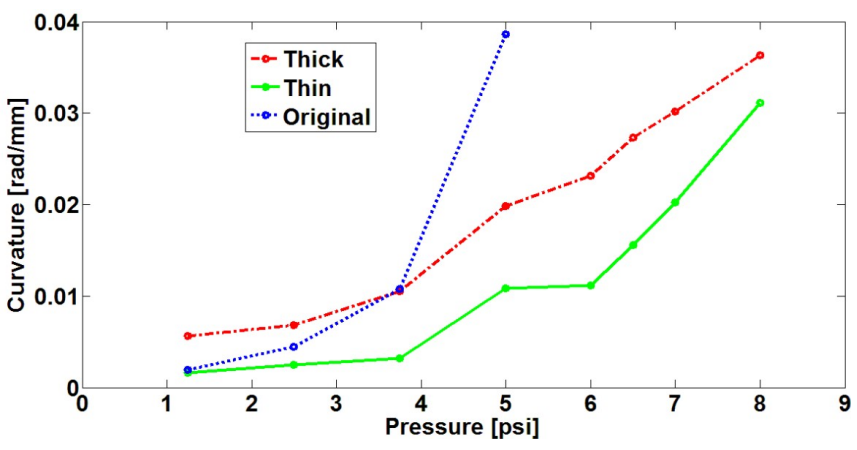

TABLE IV

THE STATIC CHANGE IN CURVATURE OF THE THREE ACTUATORS FOR THE INVESTIGATED PRESSURE INPUTS

\begin{tabular}{c||ccc}
$P_{\text {in }}[\mathrm{psi}]$ & Thick $[\mathrm{rad} / \mathrm{mm}]$ & Thin $[\mathrm{rad} / \mathrm{mm}]$ & Original $[\mathrm{rad} / \mathrm{mm}]$ \\
\hline \hline 1.25 & 0.0057 & 0.0016 & 0.0019 \\
2.5 & 0.0068 & 0.0025 & 0.0044 \\
3.75 & 0.0105 & 0.0032 & 0.0108 \\
5 & 0.0198 & 0.0108 & 0.0387 \\
6 & 0.0232 & 0.0112 & \\
6.5 & 0.0273 & 0.0155 & \\
7 & 0.0302 & 0.0203 & \\
8 & 0.0363 & 0.0311 & \\
\hline
\end{tabular}

Fig. 7. The relationships between the static bending deflection and pressure input for the three actuators.

chamber inside the soft actuators acts as a capacitance and the pneumatic tubing acts as a resistance [17] creating a single time constant.

A dynamic model is crucial for the locomotion model of the soft-robotic snake [18] to accurately simulate combinations of driving conditions to obtain improved performance. For operation as the actuation segments of a snake robot, the most significant dynamic parameter is the time constant $\tau$. Table III tabulates the time constant of all three actuators for $P_{i n}=5$ psi (as a representative and reasonable actuation pressure used in snake locomotion experiments). Other important properties listed in Table III are the height of the center-of-gravity ( $\mathrm{CoG})$, the base width, and the maximum input pressure the investigated FEAs can endure. A lower $\mathrm{CoG}$ and a wider base are preferred to improve the balance of the snake robot undulating especially on rough terrain. A higher input pressure is preferred to increase the potential force output of the segments.

Based on these numbers, the thin-and-long version of the new actuators seems to offer the best combination of the investigated properties. The only advantage of the original FEA design compared to the next generation is its higher sensitivity (larger deflection) to pressure input. In Figure 6,

it displays the highest maximum curvature values among all designs for the same pressure input values. This is due to the stiffness of the different constraint layers used in different designs. New designs use a thin plastic constraint layer, which has significantly higher bending stiffness values compared to fabric constraint used in the original design. On the other hand, it also has the highest time constants. The thin version of the new design has the smallest dynamic time constants much lower than 1 second (since it has a smaller fluidic chamber volume and hence a smaller equivalent capacitance) and it can endure pressures up to 9 psi (also observed in the thick version).

It can also be observed from Figure 7 that the static deflection of all the actuators is increasing with a non-linear function of the applied pressure and stops deforming once the actuator reaches its physical limit. These static measurements are the convergence values of dynamic responses for each pressure input, also listed in Table IV. The nonlinearity in this relation is due to stress-strain relationship of the silicone rubber elastomer. As seen in Figures 6 and 7, both of the proposed fluidic elastomer actuators offer a similar maximum deflection value to the original one, with significantly faster transient responses. 


\section{Conclusion}

We presented two kinds of soft actuators designed to overcome the drawbacks of the current segments of our soft robotic snake and provides the analysis of the dynamic features of each actuator. This work represents our effort on efficiency improvement of the snake robot by changing the mechanical design. We characterized relevant properties of the actuators and compared with the original fluidic elastomer actuator. Based on the new soft actuators the robot is expected to be more robust and be able to withstand higher input pressure (for correspondingly large output force). The large bottom surface of the new soft actuators enables a stable contact with the ground. The plastic film used as the constraint of the new actuators can be replaced with a flexible circuit in the future which means the forthcoming version of the robot will have its sensory system embedded within its body. Based on the analysis of the dynamics of the soft actuator, the parameters of the robot's mechanical design can be optimized.

The relationship between the dynamic properties and the size of the actuators should be investigated in the future in the search for the optimal dimensions for the soft snake robot. We plan to test the dynamic response of different lengths of the same type of actuator to determine the relationship. The nonlinear static response of these actuators will be modeled comprehensively.

\section{REFERENCES}

[1] T. Kamegawa, T. Yamasaki, H. Igarashi, and F. Matsuno, "Development of the snake-like rescue robot," in Robotics and Automation, 2004. Proceedings. ICRA'04. 2004 IEEE International Conference on, vol. 5. IEEE, 2004, pp. 5081-5086.

[2] A. Birk, K. Pathak, S. Schwertfeger, and W. Chonnaparamutt, "The iub rugbot: an intelligent, rugged mobile robot for search and rescue operations," in IEEE International Workshop on Safety, Security, and Rescue Robotics (SSRR). IEEE Press, vol. 10, 2006.

[3] H. Tsukagoshi, M. Sasaki, A. Kitagawa, and T. Tanaka, "Design of a higher jumping rescue robot with the optimized pneumatic drive," in Robotics and Automation, 2005. ICRA 2005. Proceedings of the 2005 IEEE International Conference on. IEEE, 2005, pp. 1276-1283.

[4] A. Crespi, A. Badertscher, A. Guignard, and A. J. Ijspeert, "Swimming and crawling with an amphibious snake robot," in Robotics and Automation, 2005. ICRA 2005. Proceedings of the 2005 IEEE International Conference on. IEEE, 2005, pp. 3024-3028.

[5] A. Crespi and A. J. Ijspeert, "Amphibot ii: An amphibious snake robot that crawls and swims using a central pattern generator," in Proceedings of the 9th international conference on climbing and walking robots (CLAWAR 2006), vol. 11, no. 7-8. Citeseer, 2006, pp. 19-27.
[6] G. Granosik and J. Borenstein, "The omnitread serpentine robot with pneumatic joint actuation," in Robot Motion and Control, 2005. RoMoCo'05. Proceedings of the Fifth International Workshop on. IEEE, 2005, pp. 105-110.

[7] K. Xu and N. Simaan, "Actuation compensation for flexible surgical snake-like robots with redundant remote actuation," in Robotics and Automation, 2006. ICRA 2006. Proceedings 2006 IEEE International Conference on. IEEE, 2006, pp. 4148-4154.

[8] N. Kamamichi, M. Yamakita, K. Asaka, and Z.-W. Luo, "A snakelike swimming robot using ipmc actuator/sensor," in Robotics and Automation, 2006. ICRA 2006. Proceedings 2006 IEEE International Conference on. IEEE, 2006, pp. 1812-1817.

[9] C. Liu and W. Liao, "A snake robot using shape memory alloys," in Robotics and Biomimetics, 2004. ROBIO 2004. IEEE International Conference on. IEEE, 2004, pp. 601-605.

[10] S. A. Morin, R. F. Shepherd, S. W. Kwok, A. A. Stokes, A. Nemiroski, and G. M. Whitesides, "Camouflage and display for soft machines," Science, vol. 337, no. 6096, pp. 828-832, 2012.

[11] B. A. Trimmer, H.-T. Lin, A. Baryshyan, G. G. Leisk, and D. L. Kaplan, "Towards a biomorphic soft robot: design constraints and solutions," in Biomedical Robotics and Biomechatronics (BioRob), 2012 4th IEEE RAS \& EMBS International Conference on. IEEE, 2012, pp. 599-605.

[12] A. Menciassi, S. Gorini, G. Pernorio, L. Weiting, F. Valvo, and P. Dario, "Design, fabrication and performances of a biomimetic robotic earthworm," in Robotics and Biomimetics, 2004. ROBIO 2004. IEEE International Conference on. IEEE, 2004, pp. 274-278.

[13] M. Calisti, M. Giorelli, G. Levy, B. Mazzolai, B. Hochner, C. Laschi, and P. Dario, "An octopus-bioinspired solution to movement and manipulation for soft robots," Bioinspiration \& biomimetics, vol. 6 , no. 3, p. 036002, 2011

[14] C. D. Onal and D. Rus, "A modular approach to soft robots," in Biomedical Robotics and Biomechatronics (BioRob), 2012 4th IEEE RAS \& EMBS International Conference on. IEEE, 2012, pp. 10381045.

[15] A. D. Marchese, C. D. Onal, and D. Rus, "Soft robot actuators using energy-efficient valves controlled by electropermanent magnets," in Intelligent Robots and Systems (IROS), 2011 IEEE/RSJ International Conference on. IEEE, 2011, pp. 756-761.

[16] C. D. Onal, X. Chen, G. M. Whitesides, and D. Rus, "Soft mobile robots with on-board chemical pressure generation," in International Symposium on Robotics Research (ISRR), 2011.

[17] C. D. Onal and D.Rus, "Autonomous undulatory serpentine locomotion utilizing body dynamics of a fluidic soft robot," Bioinspiration \& biomimetics, vol. 8, no. 2, p. 026003, 2013.

[18] M. Luo, M. Agheli, and C. D. Onal, "Theoretical modeling and experimental analysis of a pressure-operated soft robotic snake (under review)," Soft Robotics, 2014 (under review).

[19] S. Hirose, Biologically Inspired Robots: Snake-like Locomotors and Manipulators. Oxford University Press, 1993.

[20] M. Sato, M. Fukaya, and T. Iwasaki, "Serpentine locomotion with robotic snakes," Control Systems, IEEE, vol. 22, no. 1, pp. 64-81, 2002.

[21] K. Y. Pettersen, Ø. Stavdahl, and J. T. Gravdahl, Snake Robots: Modelling, Mechatronics, and Control. Springer, 2013.

[22] R. Deimel and O. Brock, "A compliant hand based on a novel pneumatic actuator," in Robotics and Automation (ICRA), 2013 IEEE International Conference on. IEEE, 2013, pp. 2047-2053. 